\title{
PSEUDOINVERSE FORMULATION OF RAYLEIGH-SCHRÖDINGER PERTURBATION THEORY FOR THE SYMMETRIC MATRIX EIGENVALUE PROBLEM
}

\author{
BRIAN J. McCARTIN
}

Received 20 March 2003

A comprehensive treatment of Rayleigh-Schrödinger perturbation theory for the symmetric matrix eigenvalue problem is furnished with emphasis on the degenerate problem. The treatment is simply based upon the Moore-Penrose pseudoinverse thus distinguishing it from alternative approaches in the literature. In addition to providing a concise matrixtheoretic formulation of this procedure, it also provides for the explicit determination of that stage of the algorithm where each higher-order eigenvector correction becomes fully determined. The theory is built up gradually with each successive stage appended with an illustrative example.

\section{Introduction}

In Rayleigh's investigation of vibrating strings with mild longitudinal density variation [9], a perturbation procedure was developed based upon the known analytical solution for a string of constant density. This technique was subsequently refined by Schrödinger [11] and applied to problems in quantum mechanics where it has become a mainstay of mathematical physics.

Mathematically, we have a discretized Laplacian-type operator embodied in a real symmetric matrix $A_{0}$, which is subjected to a small symmetric linear perturbation $A=A_{0}+\epsilon A_{1}$, due to some physical inhomogeneity. The Rayleigh-Schrödinger procedure produces approximations to the eigenvalues and eigenvectors of $A$ by a sequence of successively higher-order corrections to the eigenvalues and eigenvectors of $A_{0}$. 
The difficulty with standard treatments of this procedure [1] is that the eigenvector corrections are expressed in a form requiring the complete collection of eigenvectors of $A_{0}$. For large matrices, this is clearly an undesirable state of affairs. Consideration of the thorny issue of multiple eigenvalues of $A_{0}$ [4] only serves to exacerbate this difficulty.

This malady can be remedied by expressing the Rayleigh-Schrödinger procedure in terms of the Moore-Penrose pseudoinverse [12]. This permits these corrections to be computed knowing only the eigenvectors of $A_{0}$ corresponding to the eigenvalues of interest. In point of fact, the pseudoinverse need not be explicitly calculated since only pseudoinversevector products are required. In turn, these may be efficiently calculated by a combination of LU-factorization and orthogonal projections. However, the formalism of the pseudoinverse provides a concise formulation of the procedure and permits ready analysis of theoretical properties of the algorithm.

Since the present paper is only concerned with real symmetric matrices, the existence of a complete set of orthonormal eigenvectors is assured $[5,8,13]$. The much more difficult case for defective matrices has been considered elsewhere [7]. Moreover, we only consider the computational aspects of this procedure. Existence of the relevant perturbation expansions has been rigorously established in $[3,6,10]$.

\section{Nondegenerate case}

Consider the eigenvalue problem

$$
A x_{i}=\lambda_{i} x_{i} \quad(i=1, \ldots, n),
$$

where $A$ is a real, symmetric, $n \times n$ matrix with distinct eigenvalues $\lambda_{i}$ $(i=1, \ldots, n)$ and, consequently, orthogonal eigenvectors $x_{i}(i=1, \ldots, n)$. Furthermore,

$$
A(\epsilon)=A_{0}+\epsilon A_{1}
$$

where $A_{0}$ is likewise real and symmetric but may possess multiple eigenvalues (called degeneracies in the physics literature). Any attempt to drop the assumption on the eigenstructure of $A$ leads to a RayleighSchrödinger iteration that never terminates [3, page 92]. In this section, we consider the nondegenerate case where the unperturbed eigenvalues $\lambda_{i}^{(0)}(i=1, \ldots, n)$ are all distinct. Consideration of the degenerate case is deferred to the next section. 
Under these assumptions, it is shown in $[3,6,10]$ that the eigenvalues and eigenvectors of $A$ possess the respective perturbation expansions

$$
\lambda_{i}(\epsilon)=\sum_{k=0}^{\infty} \epsilon^{k} \lambda_{i}^{(k)}, \quad x_{i}(\epsilon)=\sum_{k=0}^{\infty} \epsilon^{k} x_{i}^{(k)} \quad(i=1, \ldots, n)
$$

for sufficiently small $\epsilon$. Clearly, the zeroth-order terms $\left\{\lambda_{i}^{(0)} ; x_{i}^{(0)}\right\}(i=$ $1, \ldots, n)$ are the eigenpairs of the unperturbed matrix $A_{0}$. That is,

$$
\left(A_{0}-\lambda_{i}^{(0)} I\right) x_{i}^{(0)}=0 \quad(i=1, \ldots, n)
$$

The unperturbed mutually orthogonal eigenvectors $x_{i}^{(0)}(i=1, \ldots, n)$ are assumed to have been normalized to unity.

Substitution of (2.2) and (2.3) into (2.1) yields the recurrence relation

$$
\begin{aligned}
\left(A_{0}-\lambda_{i}^{(0)} I\right) x_{i}^{(k)}= & -\left(A_{1}-\lambda_{i}^{(1)} I\right) x_{i}^{(k-1)} \\
& +\sum_{j=0}^{k-2} \lambda_{i}^{(k-j)} x_{i}^{(j)} \quad(k=1, \ldots, \infty ; i=1, \ldots, n) .
\end{aligned}
$$

For fixed $i$, solvability of (2.5) requires that its right-hand side be orthogonal to $\left\{x_{l}^{(0)}\right\}_{l=1}^{n}$ for all $k$. Thus, the value of $x_{i}^{(j)}$ determines $\lambda_{i}^{(j+1)}$. Specifically,

$$
\lambda_{i}^{(j+1)}=\left\langle x_{i}^{(0)}, A_{1} x_{i}^{(j)}\right\rangle
$$

where we have employed the so-called intermediate normalization that $x_{i}^{(k)}$ will be chosen to be orthogonal to $x_{i}^{(0)}$ for $k=1, \ldots, \infty$. This is equivalent to $\left\langle x_{i}^{(0)}, x_{i}(\epsilon)\right\rangle=1$ and this normalization will be used throughout the remainder of this paper.

A beautiful result due to Dalgarno and Stewart [2], sometimes incorrectly attributed to Wigner in the physics literature, says that much more is true: the value of the eigenvector correction $x_{i}^{(j)}$, in fact, determines the eigenvalues through $\lambda_{i}^{(2 j+1)}$. Within the present framework, this may be established by the following constructive procedure which heavily exploits the symmetry of $A_{0}$ and $A_{1}$. 
462 Pseudoinverse formulation of perturbation theory

We commence by observing that

$$
\begin{aligned}
\lambda_{i}^{(k)} & =\left\langle x_{i}^{(0)},\left(A_{1}-\lambda_{i}^{(1)} I\right) x_{i}^{(k-1)}\right\rangle=\left\langle x_{i}^{(k-1)},\left(A_{1}-\lambda_{i}^{(1)} I\right) x_{i}^{(0)}\right\rangle \\
& =-\left\langle x_{i}^{(k-1)},\left(A_{0}-\lambda_{i}^{(0)} I\right) x_{i}^{(1)}\right\rangle=-\left\langle x_{i}^{(1)},\left(A_{0}-\lambda_{i}^{(0)} I\right) x_{i}^{(k-1)}\right\rangle \\
& =\left\langle x_{i}^{(1)},\left(A_{1}-\lambda_{i}^{(1)} I\right) x_{i}^{(k-2)}\right\rangle-\sum_{l=2}^{k-1} \lambda_{i}^{(l)}\left\langle x_{i}^{(1)}, x_{i}^{(k-1-l)}\right\rangle .
\end{aligned}
$$

Continuing in this fashion, we eventually arrive at, for even $k=2 j$,

$$
\begin{aligned}
\lambda_{i}^{(2 j)}= & \left\langle x_{i}^{(j-1)},\left(A_{1}-\lambda_{i}^{(1)} I\right) x_{i}^{(j)}\right\rangle-\sum_{\mu=2}^{j-1} \lambda_{i}^{(\mu)} \sum_{v=j-\mu+1}^{j}\left\langle x_{i}^{(2 j-\mu-v)}, x_{i}^{(v)}\right\rangle \\
& -\sum_{\mu=j}^{2 j-2} \lambda_{i}^{(\mu)} \sum_{\nu=1}^{2 j-\mu-1}\left\langle x_{i}^{(2 j-\mu-v)}, x_{i}^{(v)}\right\rangle,
\end{aligned}
$$

while, for odd $k=2 j+1$,

$$
\begin{aligned}
\lambda_{i}^{(2 j+1)}= & \left\langle x_{i}^{(j)},\left(A_{1}-\lambda_{i}^{(1)} I\right) x_{i}^{(j)}\right\rangle-\sum_{\mu=2}^{j-1} \lambda_{i}^{(\mu)} \sum_{v=j-\mu+1}^{j}\left\langle x_{i}^{(2 j-\mu-v+1)}, x_{i}^{(v)}\right\rangle \\
& -\sum_{\mu=j}^{2 j-1} \lambda_{i}^{(\mu)} \sum_{\nu=1}^{2 j-\mu}\left\langle x_{i}^{(2 j-\mu-v+1)}, x_{i}^{(v)}\right\rangle .
\end{aligned}
$$

This important pair of equations will henceforth be referred to as the Dalgarno-Stewart identities.

The eigenfunction corrections are determined recursively from (2.5) as

$$
\begin{gathered}
x_{i}^{(k)}=\left(A_{0}-\lambda_{i}^{(0)} I\right)^{\dagger}\left[-\left(A_{1}-\lambda_{i}^{(1)} I\right) x_{i}^{(k-1)}+\sum_{j=0}^{k-2} \lambda_{i}^{(k-j)} x_{i}^{(j)}\right] \\
(k=1, \ldots, \infty ; i=1, \ldots, n),
\end{gathered}
$$

where $\left(A_{0}-\lambda_{i}^{(0)} I\right)^{\dagger}$ denotes the Moore-Penrose pseudoinverse [12] of $\left(A_{0}-\lambda_{i}^{(0)} I\right)$ and intermediate normalization has been employed.

Example 2.1. Define

$$
A_{0}=\left[\begin{array}{lll}
1 & 0 & 0 \\
0 & 1 & 0 \\
0 & 0 & 2
\end{array}\right], \quad A_{1}=\left[\begin{array}{lll}
1 & 1 & 1 \\
1 & 1 & 0 \\
1 & 0 & 0
\end{array}\right]
$$


Using Matlab's Symbolic Toolbox, we find that

$$
\begin{aligned}
& \lambda_{1}(\epsilon)=1-\frac{1}{2} \epsilon^{2}-\frac{1}{8} \epsilon^{3}+\frac{1}{4} \epsilon^{4}+\frac{25}{128} \epsilon^{5}+\cdots, \\
& \lambda_{2}(\epsilon)=1+2 \epsilon-\frac{1}{2} \epsilon^{2}-\frac{7}{8} \epsilon^{3}-\frac{5}{4} \epsilon^{4}-\frac{153}{128} \epsilon^{5}+\cdots, \\
& \lambda_{3}(\epsilon)=2+\epsilon^{2}+\epsilon^{3}+\epsilon^{4}+\epsilon^{5}+\cdots .
\end{aligned}
$$

Applying the nondegenerate Rayleigh-Schrödinger procedure developed above to

$$
\lambda_{3}^{(0)}=2, \quad x_{3}^{(0)}=\left[\begin{array}{l}
0 \\
0 \\
1
\end{array}\right]
$$

we arrive at

$$
\lambda_{3}^{(1)}=\left\langle x_{3}^{(0)}, A_{1} x_{3}^{(0)}\right\rangle=0 .
$$

Solving

$$
\left(A_{0}-\lambda_{3}^{(0)} I\right) x_{3}^{(1)}=-\left(A_{1}-\lambda_{3}^{(1)} I\right) x_{3}^{(0)}
$$

produces

$$
x_{3}^{(1)}=\left[\begin{array}{l}
1 \\
0 \\
0
\end{array}\right] \text {. }
$$

In turn, this produces

$$
\lambda_{3}^{(2)}=\left\langle x_{3}^{(0)}, A_{1} x_{3}^{(1)}\right\rangle=1,
$$

while the Dalgarno-Stewart identities yield

$$
\lambda_{3}^{(3)}=\left\langle x_{3}^{(1)},\left(A_{1}-\lambda_{3}^{(1)} I\right) x_{3}^{(1)}\right\rangle=1 .
$$

Solving

$$
\left(A_{0}-\lambda_{3}^{(0)} I\right) x_{3}^{(2)}=-\left(A_{1}-\lambda_{3}^{(1)} I\right) x_{3}^{(1)}+\lambda_{3}^{(2)} x_{3}^{(0)}
$$


464 Pseudoinverse formulation of perturbation theory

produces

$$
x_{3}^{(2)}=\left[\begin{array}{l}
1 \\
1 \\
0
\end{array}\right] \text {. }
$$

Again, the Dalgarno-Stewart identities yield

$$
\begin{aligned}
& \lambda_{3}^{(4)}=\left\langle x_{3}^{(1)},\left(A_{1}-\lambda_{3}^{(1)} I\right) x_{3}^{(2)}\right\rangle-\lambda_{3}^{(2)}\left\langle x_{3}^{(1)}, x_{3}^{(1)}\right\rangle=1, \\
& \lambda_{3}^{(5)}=\left\langle x_{3}^{(2)},\left(A_{1}-\lambda_{3}^{(1)} I\right) x_{3}^{(2)}\right\rangle-2 \lambda_{3}^{(2)}\left\langle x_{3}^{(2)}, x_{3}^{(1)}\right\rangle-\lambda_{3}^{(3)}\left\langle x_{3}^{(1)}, x_{3}^{(1)}\right\rangle=1 .
\end{aligned}
$$

\section{Degenerate case}

When $A_{0}$ possesses multiple eigenvalues (the so-called degenerate case), the above straightforward analysis for the nondegenerate case encounters serious complications. This is a consequence of the fact that, in this new case, Rellich's theorem [10, pages 42-45] guarantees the existence of the perturbation expansions (2.3) only for certain special unperturbed eigenvectors. These special unperturbed eigenvectors cannot be specified a priori but must instead emerge from the perturbation procedure itself.

Furthermore, the higher-order corrections to these special unperturbed eigenvectors are more stringently constrained than previously since they must be chosen so that (2.5) is always solvable. That is, they must be chosen so that the right-hand side of (2.5) is always orthogonal to the entire eigenspace associated with the multiple eigenvalue in question.

Thus, without any loss of generality, suppose that $\lambda_{1}^{(0)}=\lambda_{2}^{(0)}=\cdots=$ $\lambda_{m}^{(0)}=\lambda^{(0)}$ is just such an eigenvalue of multiplicity $m$ with corresponding known orthonormal eigenvectors $x_{1}^{(0)}, x_{2}^{(0)}, \ldots, x_{m}^{(0)}$. Then, we are required to determine appropriate linear combinations

$$
y_{i}^{(0)}=a_{1}^{(i)} x_{1}^{(0)}+a_{2}^{(i)} x_{2}^{(0)}+\cdots+a_{m}^{(i)} x_{m}^{(0)} \quad(i=1, \ldots, m)
$$

so that the expansions (2.3) are valid with $x_{i}^{(k)}$ replaced by $y_{i}^{(k)}$. In point of fact, the remainder of this paper will assume that $x_{i}$ has been replaced by $y_{i}$ in (2.3)-(2.10). Moreover, the higher-order eigenvector corrections $y_{i}^{(k)}$ must be suitably determined. Since we would like $\left\{y_{i}^{(0)}\right\}_{i=1}^{m}$ to be likewise orthonormal, we require that

$$
a_{1}^{(\mu)} a_{1}^{(v)}+a_{2}^{(\mu)} a_{2}^{(v)}+\cdots+a_{m}^{(\mu)} a_{m}^{(v)}=\delta_{\mu, v}
$$


Recall that we have assumed throughout that the perturbed matrix $A(\epsilon)$ itself has distinct eigenvalues, so that eventually all such degeneracies will be fully resolved. What significantly complicates matters is that it is not known beforehand at what stages portions of the degeneracy will be resolved.

In order to bring order to a potentially calamitous situation, we will first begin by considering the case where the degeneracy is fully resolved at first order. Only then do we move on to study the case where the degeneracy is completely and simultaneously resolved at second order. This will pave the way for the treatment of $N$ th order degeneracy resolution. Finally, we will have laid sufficient groundwork to permit treatment of the most general case of mixed degeneracy where resolution occurs across several different orders. Each stage in this process will be concluded with an illustrative example. This seems preferable to presenting an impenetrable collection of opaque formulae.

\subsection{First-order degeneracy}

We first dispense with the case of first-order degeneracy wherein $\lambda_{i}^{(1)}$ $(i=1, \ldots, m)$ are all distinct. In this event, we determine $\left\{\lambda_{i}^{(1)} ; y_{i}^{(0)}\right\}_{i=1}^{m}$ by insisting that (2.5) be solvable for $k=1$ and $i=1, \ldots, m$. In order for this to obtain, it is both necessary and sufficient that, for each fixed $i$,

$$
\left\langle x_{\mu}^{(0)},\left(A_{1}-\lambda_{i}^{(1)} I\right) y_{i}^{(0)}\right\rangle=0 \quad(\mu=1, \ldots, m) .
$$

Inserting (3.1) and invoking the orthonormality of $\left\{x_{\mu}^{(0)}\right\}_{\mu=1}^{m}$, we arrive at, in matrix form,

$$
\left[\begin{array}{ccc}
\left\langle x_{1}^{(0)}, A_{1} x_{1}^{(0)}\right\rangle & \cdots & \left\langle x_{1}^{(0)}, A_{1} x_{m}^{(0)}\right\rangle \\
\vdots & \ddots & \vdots \\
\left\langle x_{m}^{(0)}, A_{1} x_{1}^{(0)}\right\rangle & \cdots & \left\langle x_{m}^{(0)}, A_{1} x_{m}^{(0)}\right\rangle
\end{array}\right]\left[\begin{array}{c}
a_{1}^{(i)} \\
\vdots \\
a_{m}^{(i)}
\end{array}\right]=\lambda_{i}^{(1)}\left[\begin{array}{c}
a_{1}^{(i)} \\
\vdots \\
a_{m}^{(i)}
\end{array}\right]
$$

Thus, each $\lambda_{i}^{(1)}$ is an eigenvalue with corresponding eigenvector $\left[a_{1}^{(i)}, \ldots\right.$, $\left.a_{m}^{(i)}\right]^{T}$ of the matrix $M$ defined by $M_{\mu, v}=\left\langle x_{\mu}^{(0)}, M^{(1)} x_{v}^{(0)}\right\rangle(\mu, v=1, \ldots, m)$, where $M^{(1)}:=A_{1}$.

By assumption, the symmetric matrix $M$ has $m$ distinct real eigenvalues and hence orthonormal eigenvectors described by (3.2). These, in turn, may be used in concert with (3.1) to yield the desired special unperturbed eigenvectors alluded to above.

Now that $\left\{y_{i}^{(0)}\right\}_{i=1}^{m}$ are fully determined, we have by (2.6) the identities

$$
\lambda_{i}^{(1)}=\left\langle y_{i}^{(0)}, A_{1} y_{i}^{(0)}\right\rangle \quad(i=1, \ldots, m) .
$$


466 Pseudoinverse formulation of perturbation theory

Furthermore, the combination of (3.2) and (3.4) yields

$$
\left\langle y_{i}^{(0)}, A_{1} y_{j}^{(0)}\right\rangle=0 \quad(i \neq j)
$$

The remaining eigenvalue corrections $\lambda_{i}^{(k)}(k \geq 2)$ may be obtained from the Dalgarno-Stewart identities.

Whenever (2.5) is solvable, we will express its solution as

$$
y_{i}^{(k)}=\hat{y}_{i}^{(k)}+\beta_{1, k}^{(i)} y_{1}^{(0)}+\beta_{2, k}^{(i)} y_{2}^{(0)}+\cdots+\beta_{m, k}^{(i)} y_{m}^{(0)} \quad(i=1, \ldots, m),
$$

where $\hat{y}_{i}^{(k)}:=\left(A_{0}-\lambda^{(0)} I\right)^{\dagger}\left[-\left(A_{1}-\lambda_{i}^{(1)} I\right) y_{i}^{(k-1)}+\sum_{j=0}^{k-2} \lambda_{i}^{(k-j)} y_{i}^{(j)}\right]$ has no components in the $\left\{y_{j}^{(0)}\right\}_{j=1}^{m}$ directions. In the light of intermediate normalization, we have $\beta_{i, k}^{(i)}=0(i=1, \ldots, m)$. Furthermore, $\beta_{j, k}^{(i)}(i \neq j)$ are to be determined from the condition that (2.5) be solvable for $k \leftarrow k+1$ and $i=1, \ldots, m$.

Since, by design, (2.5) is solvable for $k=1$, we may proceed recursively. After considerable algebraic manipulation, the end result is

$$
\beta_{j, k}^{(i)}=\frac{\left\langle y_{j}^{(0)}, A_{1} \hat{y}_{i}^{(k)}\right\rangle-\sum_{l=1}^{k-1} \lambda_{i}^{(k-l+1)} \beta_{j, l}^{(i)}}{\lambda_{i}^{(1)}-\lambda_{j}^{(1)}} \quad(i \neq j) .
$$

The existence of this formula guarantees that each $y_{i}^{(k)}$ is uniquely determined by enforcing solvability of (2.5) for $k \leftarrow k+1$.

Example 3.1. We resume with Example 2.1 and the first-order degeneracy between $\lambda_{1}^{(0)}$ and $\lambda_{2}^{(0)}$. With the choice

$$
x_{1}^{(0)}=\left[\begin{array}{l}
1 \\
0 \\
0
\end{array}\right], \quad x_{2}^{(0)}=\left[\begin{array}{l}
0 \\
1 \\
0
\end{array}\right],
$$

we have

$$
M=\left[\begin{array}{ll}
1 & 1 \\
1 & 1
\end{array}\right]
$$

with eigenpairs

$$
\lambda_{1}^{(1)}=0, \quad\left[\begin{array}{l}
a_{1}^{(1)} \\
a_{2}^{(1)}
\end{array}\right]=\left[\begin{array}{c}
\frac{1}{\sqrt{2}} \\
-\frac{1}{\sqrt{2}}
\end{array}\right], \quad \lambda_{2}^{(1)}=2, \quad\left[\begin{array}{l}
a_{1}^{(2)} \\
a_{2}^{(2)}
\end{array}\right]=\left[\begin{array}{c}
\frac{1}{\sqrt{2}} \\
\frac{1}{\sqrt{2}}
\end{array}\right] .
$$


Availing ourselves of (3.1), the special unperturbed eigenvectors are now fully determined as

$$
y_{1}^{(0)}=\left[\begin{array}{c}
\frac{1}{\sqrt{2}} \\
-\frac{1}{\sqrt{2}} \\
0
\end{array}\right], \quad y_{2}^{(0)}=\left[\begin{array}{c}
\frac{1}{\sqrt{2}} \\
\frac{1}{\sqrt{2}} \\
0
\end{array}\right] \text {. }
$$

Solving (2.5), for $k=1$,

$$
\left(A_{0}-\lambda^{(0)} I\right) y_{i}^{(1)}=-\left(A_{1}-\lambda_{i}^{(1)} I\right) y_{i}^{(0)} \quad(i=1,2),
$$

produces

$$
y_{1}^{(1)}=\left[\begin{array}{c}
a \\
a \\
-\frac{1}{\sqrt{2}}
\end{array}\right], \quad y_{2}^{(1)}=\left[\begin{array}{c}
b \\
-b \\
-\frac{1}{\sqrt{2}}
\end{array}\right]
$$

where we have invoked intermediate normalization. Observe that, unlike the nondegenerate case, $y_{i}^{(1)}(i=1,2)$ are not yet fully determined.

We next enforce solvability of (2.5) for $k=2$ :

$$
\left\langle y_{j}^{(0)},-\left(A_{1}-\lambda_{i}^{(1)} I\right) y_{i}^{(1)}+\lambda_{i}^{(2)} y_{i}^{(0)}\right\rangle=0 \quad(i \neq j),
$$

thereby producing

$$
y_{1}^{(1)}=\left[\begin{array}{c}
\frac{1}{4 \sqrt{2}} \\
\frac{1}{4 \sqrt{2}} \\
-\frac{1}{\sqrt{2}}
\end{array}\right], \quad y_{2}^{(1)}=\left[\begin{array}{c}
-\frac{1}{4 \sqrt{2}} \\
\frac{1}{4 \sqrt{2}} \\
-\frac{1}{\sqrt{2}}
\end{array}\right] \text {. }
$$

With $y_{i}^{(1)}(i=1,2)$ now fully determined, the Dalgarno-Stewart identities yield

$$
\begin{array}{ll}
\lambda_{1}^{(2)}=\left\langle y_{1}^{(0)}, A_{1} y_{1}^{(1)}\right\rangle=-\frac{1}{2} ; \quad & \lambda_{1}^{(3)}=\left\langle y_{1}^{(1)},\left(A_{1}-\lambda_{1}^{(1)} I\right) y_{1}^{(1)}\right\rangle=-\frac{1}{8} \\
\lambda_{2}^{(2)}=\left\langle y_{2}^{(0)}, A_{1} y_{2}^{(1)}\right\rangle=-\frac{1}{2} ; & \lambda_{2}^{(3)}=\left\langle y_{2}^{(1)},\left(A_{1}-\lambda_{2}^{(1)} I\right) y_{2}^{(1)}\right\rangle=-\frac{7}{8}
\end{array}
$$


Solving (2.5), for $k=2$,

$$
\left(A_{0}-\lambda^{(0)} I\right) y_{i}^{(2)}=-\left(A_{1}-\lambda_{i}^{(1)} I\right) y_{i}^{(1)}+\lambda_{i}^{(2)} y_{i}^{(0)} \quad(i=1,2),
$$

produces

$$
y_{1}^{(2)}=\left[\begin{array}{c}
c \\
c \\
-\frac{1}{4 \sqrt{2}}
\end{array}\right], \quad y_{2}^{(2)}=\left[\begin{array}{c}
d \\
-d \\
-\frac{7}{4 \sqrt{2}}
\end{array}\right],
$$

where we have again invoked intermediate normalization. Once again observe that, unlike the nondegenerate case, $y_{i}^{(2)}(i=1,2)$ are not yet fully determined.

We now enforce solvability of (2.5) for $k=3$ :

$$
\left\langle y_{j}^{(0)},-\left(A_{1}-\lambda_{i}^{(1)} I\right) y_{i}^{(2)}+\lambda_{i}^{(2)} y_{i}^{(1)}+\lambda_{i}^{(3)} y_{i}^{(0)}\right\rangle=0 \quad(i \neq j),
$$

thereby fully determining

$$
y_{1}^{(2)}=\left[\begin{array}{c}
0 \\
0 \\
-\frac{1}{4 \sqrt{2}}
\end{array}\right], \quad y_{2}^{(2)}=\left[\begin{array}{c}
-\frac{1}{2 \sqrt{2}} \\
\frac{1}{2 \sqrt{2}} \\
-\frac{7}{4 \sqrt{2}}
\end{array}\right] .
$$

Subsequent application of the Dalgarno-Stewart identities yields

$$
\begin{aligned}
& \lambda_{1}^{(4)}=\left\langle y_{1}^{(1)},\left(A_{1}-\lambda_{1}^{(1)} I\right) y_{1}^{(2)}\right\rangle-\lambda_{1}^{(2)}\left\langle y_{1}^{(1)}, y_{1}^{(1)}\right\rangle=\frac{1}{4}, \\
& \lambda_{1}^{(5)}=\left\langle y_{1}^{(2)},\left(A_{1}-\lambda_{1}^{(1)} I\right) y_{1}^{(2)}\right\rangle-2 \lambda_{1}^{(2)}\left\langle y_{1}^{(2)}, y_{1}^{(1)}\right\rangle-\lambda_{1}^{(3)}\left\langle y_{1}^{(1)}, y_{1}^{(1)}\right\rangle=\frac{25}{128}, \\
& \lambda_{2}^{(4)}=\left\langle y_{2}^{(1)},\left(A_{1}-\lambda_{2}^{(1)} I\right) y_{2}^{(2)}\right\rangle-\lambda_{2}^{(2)}\left\langle y_{2}^{(1)}, y_{2}^{(1)}\right\rangle=-\frac{5}{4}, \\
& \lambda_{2}^{(5)}=\left\langle y_{2}^{(2)},\left(A_{1}-\lambda_{2}^{(1)} I\right) y_{2}^{(2)}\right\rangle-2 \lambda_{2}^{(2)}\left\langle y_{2}^{(2)}, y_{2}^{(1)}\right\rangle-\lambda_{2}^{(3)}\left\langle y_{2}^{(1)}, y_{2}^{(1)}\right\rangle=-\frac{153}{128}
\end{aligned}
$$

\subsection{Second-order degeneracy}

We next consider the case of second-order degeneracy which is characterized by the conditions $\lambda_{1}^{(0)}=\lambda_{2}^{(0)}=\cdots=\lambda_{m}^{(0)}=\lambda^{(0)}$ and $\lambda_{1}^{(1)}=\lambda_{2}^{(1)}=\cdots=$ $\lambda_{m}^{(1)}=\lambda^{(1)}$, while $\lambda_{i}^{(2)}(i=1, \ldots, m)$ are all distinct. Thus, even though $\lambda^{(1)}$ 
is obtained as the only eigenvalue of (3.4), $\left\{y_{i}^{(0)}\right\}_{i=1}^{m}$ are still indeterminate after enforcing solvability of (2.5) for $k=1$.

Hence, we will determine $\left\{\lambda_{i}^{(2)} ; y_{i}^{(0)}\right\}_{i=1}^{m}$ by insisting that (2.5) be solvable for $k=2$ and $i=1, \ldots, m$. This requirement is equivalent to the condition that, for each fixed $i$,

$$
\left\langle x_{\mu}^{(0)},-\left(A_{1}-\lambda^{(1)} I\right) y_{i}^{(1)}+\lambda_{i}^{(2)} y_{i}^{(0)}\right\rangle=0 \quad(\mu=1, \ldots, m) .
$$

Inserting (3.1) as well as (3.7) with $k=1$ and invoking the orthonormality of $\left\{x_{\mu}^{(0)}\right\}_{\mu=1}^{m}$, we arrive at, in matrix form,

$$
\left[\begin{array}{ccc}
\left\langle x_{1}^{(0)}, M^{(2)} x_{1}^{(0)}\right\rangle & \cdots & \left\langle x_{1}^{(0)}, M^{(2)} x_{m}^{(0)}\right\rangle \\
\vdots & \ddots & \vdots \\
\left\langle x_{m}^{(0)}, M^{(2)} x_{1}^{(0)}\right\rangle & \cdots & \left\langle x_{m}^{(0)}, M^{(2)} x_{m}^{(0)}\right\rangle
\end{array}\right]\left[\begin{array}{c}
a_{1}^{(i)} \\
\vdots \\
a_{m}^{(i)}
\end{array}\right]=\lambda_{i}^{(2)}\left[\begin{array}{c}
a_{1}^{(i)} \\
\vdots \\
a_{m}^{(i)}
\end{array}\right]
$$

where $M^{(2)}:=-\left(A_{1}-\lambda^{(1)} I\right)\left(A_{0}-\lambda^{(0)} I\right)^{\dagger}\left(A_{1}-\lambda^{(1)} I\right)$. Thus, each $\lambda_{i}^{(2)}$ is an eigenvalue with corresponding eigenvector $\left[a_{1}^{(i)}, \ldots, a_{m}^{(i)}\right]^{T}$ of the matrix $M$ defined by $M_{\mu, v}=\left\langle x_{\mu}^{(0)}, M^{(2)} x_{v}^{(0)}\right\rangle(\mu, v=1, \ldots, m)$.

By assumption, the symmetric matrix $M$ has $m$ distinct real eigenvalues and hence orthonormal eigenvectors described by (3.2). These, in turn, may be used in concert with (3.1) to yield the desired special unperturbed eigenvectors alluded to above.

Now that $\left\{y_{i}^{(0)}\right\}_{i=1}^{m}$ are fully determined, we have by the combination of (3.2) and (3.24) the identities

$$
\left\langle y_{i}^{(0)}, M^{(2)} y_{j}^{(0)}\right\rangle=\lambda_{i}^{(2)} \cdot \delta_{i, j}
$$

The remaining eigenvalue corrections $\lambda_{i}^{(k)}(k \geq 3)$ may be obtained from the Dalgarno-Stewart identities.

Analogous to the case of first-order degeneracy, $\beta_{j, k}^{(i)}(i \neq j)$ of (3.7) are to be determined from the condition that (2.5) be solvable for $k \leftarrow k+2$ and $i=1, \ldots, m$. Since, by design, (2.5) is solvable for $k=1,2$, we may proceed recursively. After considerable algebraic manipulation, the end result is

$$
\beta_{j, k}^{(i)}=\frac{\left\langle y_{j}^{(0)}, M^{(2)} \hat{y}_{i}^{(k)}\right\rangle-\sum_{l=1}^{k-1} \lambda_{i}^{(k-l+2)} \beta_{j, l}^{(i)}}{\lambda_{i}^{(2)}-\lambda_{j}^{(2)}} \quad(i \neq j) .
$$


The existence of this formula guarantees that each $y_{i}^{(k)}$ is uniquely determined by enforcing solvability of (2.5) for $k \leftarrow k+2$.

Example 3.2. Define

$$
A_{0}=\left[\begin{array}{lllll}
1 & 0 & 0 & 0 & 0 \\
0 & 1 & 0 & 0 & 0 \\
0 & 0 & 1 & 0 & 0 \\
0 & 0 & 0 & 2 & 0 \\
0 & 0 & 0 & 0 & 3
\end{array}\right], \quad A_{1}=\left[\begin{array}{lllll}
1 & 0 & 0 & 1 & 0 \\
0 & 1 & 0 & 0 & 1 \\
0 & 0 & 1 & 0 & 0 \\
1 & 0 & 0 & 0 & 0 \\
0 & 1 & 0 & 0 & 0
\end{array}\right] .
$$

Using Matlab's Symbolic Toolbox, we find that

$$
\begin{aligned}
& \lambda_{1}(\epsilon)=1+\epsilon, \\
& \lambda_{2}(\epsilon)=1+\epsilon-\frac{1}{2} \epsilon^{2}-\frac{1}{4} \epsilon^{3}+\frac{1}{8} \epsilon^{5}+\cdots, \\
& \lambda_{3}(\epsilon)=1+\epsilon-\epsilon^{2}-\epsilon^{3}+2 \epsilon^{5}+\cdots, \\
& \lambda_{4}(\epsilon)=2+\epsilon^{2}+\epsilon^{3}-2 \epsilon^{5}+\cdots \\
& \lambda_{5}(\epsilon)=3+\frac{1}{2} \epsilon^{2}+\frac{1}{4} \epsilon^{3}-\frac{1}{8} \epsilon^{5}+\cdots
\end{aligned}
$$

We focus on the second-order degeneracy amongst $\lambda_{1}^{(0)}=\lambda_{2}^{(0)}=\lambda_{3}^{(0)}=$ $\lambda^{(0)}=1$. With the choice

$$
x_{1}^{(0)}=\left[\begin{array}{l}
1 \\
0 \\
0 \\
0 \\
0
\end{array}\right], \quad x_{2}^{(0)}=\left[\begin{array}{l}
0 \\
1 \\
0 \\
0 \\
0
\end{array}\right], \quad x_{3}^{(0)}=\left[\begin{array}{l}
0 \\
0 \\
1 \\
0 \\
0
\end{array}\right],
$$

we have from (3.4), which enforces solvability of (2.5) for $k=1$,

$$
M=\left[\begin{array}{lll}
1 & 0 & 0 \\
0 & 1 & 0 \\
0 & 0 & 1
\end{array}\right]
$$

with triple eigenvalue $\lambda^{(1)}=1$.

Moving on to (3.24), which enforces solvability of (2.5) for $k=2$, we have

$$
M=\left[\begin{array}{ccc}
-1 & 0 & 0 \\
0 & -\frac{1}{2} & 0 \\
0 & 0 & 0
\end{array}\right]
$$


with eigenpairs

$$
\begin{aligned}
& \lambda_{1}^{(2)}=0, \quad\left[\begin{array}{l}
a_{1}^{(1)} \\
a_{2}^{(1)} \\
a_{3}^{(1)}
\end{array}\right]=\left[\begin{array}{l}
0 \\
0 \\
1
\end{array}\right] ; \\
& \lambda_{2}^{(2)}=-\frac{1}{2}, \quad\left[\begin{array}{l}
a_{1}^{(2)} \\
a_{2}^{(2)} \\
a_{3}^{(2)}
\end{array}\right]=\left[\begin{array}{l}
0 \\
1 \\
0
\end{array}\right] ; \\
& \lambda_{3}^{(2)}=-1, \quad\left[\begin{array}{l}
a_{1}^{(3)} \\
a_{2}^{(3)} \\
a_{3}^{(3)}
\end{array}\right]=\left[\begin{array}{l}
1 \\
0 \\
0
\end{array}\right] .
\end{aligned}
$$

Availing ourselves of (3.1), the special unperturbed eigenvectors are now fully determined as

$$
y_{1}^{(0)}=\left[\begin{array}{l}
0 \\
0 \\
1 \\
0 \\
0
\end{array}\right], \quad y_{2}^{(0)}=\left[\begin{array}{l}
0 \\
1 \\
0 \\
0 \\
0
\end{array}\right], \quad y_{3}^{(0)}=\left[\begin{array}{l}
1 \\
0 \\
0 \\
0 \\
0
\end{array}\right] \text {. }
$$

Solving (2.5), for $k=1$,

$$
\left(A_{0}-\lambda^{(0)} I\right) y_{i}^{(1)}=-\left(A_{1}-\lambda^{(1)} I\right) y_{i}^{(0)} \quad(i=1,2,3),
$$

produces

$$
y_{1}^{(1)}=\left[\begin{array}{c}
\alpha_{1} \\
\beta_{1} \\
0 \\
0 \\
0
\end{array}\right], \quad y_{2}^{(1)}=\left[\begin{array}{c}
\alpha_{2} \\
0 \\
\gamma_{2} \\
0 \\
1 \\
-\frac{1}{2}
\end{array}\right], \quad y_{3}^{(1)}=\left[\begin{array}{c}
0 \\
\beta_{3} \\
\gamma_{3} \\
-1 \\
0
\end{array}\right]
$$

where we have invoked intermediate normalization. Observe that $y_{i}^{(1)}$ $(i=1,2,3)$ are not yet fully determined.

Solving (2.5), for $k=2$,

$$
\left(A_{0}-\lambda^{(0)} I\right) y_{i}^{(2)}=-\left(A_{1}-\lambda^{(1)} I\right) y_{i}^{(1)}+\lambda_{i}^{(2)} y_{i}^{(0)} \quad(i=1,2,3),
$$


472 Pseudoinverse formulation of perturbation theory

produces

$$
y_{1}^{(2)}=\left[\begin{array}{c}
a_{1} \\
b_{1} \\
0 \\
-\alpha_{1} \\
-\frac{\beta_{1}}{2}
\end{array}\right], \quad y_{2}^{(2)}=\left[\begin{array}{c}
a_{2} \\
0 \\
c_{2} \\
-\alpha_{2} \\
1 \\
-\frac{1}{4}
\end{array}\right], \quad y_{3}^{(2)}=\left[\begin{array}{c}
0 \\
b_{3} \\
c_{3} \\
-1 \\
-\frac{\beta_{3}}{2}
\end{array}\right] \text {, }
$$

where we have invoked intermediate normalization. Likewise, $y_{i}^{(2)}(i=$ $1,2,3)$ are not yet fully determined.

We next enforce solvability of (2.5) for $k=3$ :

$$
\left\langle y_{j}^{(0)},-\left(A_{1}-\lambda^{(1)} I\right) y_{i}^{(2)}+\lambda_{i}^{(2)} y_{i}^{(1)}+\lambda_{i}^{(3)} y_{i}^{(0)}\right\rangle=0 \quad(i \neq j),
$$

thereby producing

$$
\begin{aligned}
& y_{1}^{(1)}=\left[\begin{array}{l}
0 \\
0 \\
0 \\
0 \\
0
\end{array}\right] ; \quad y_{2}^{(1)}=\left[\begin{array}{c}
0 \\
0 \\
0 \\
0 \\
-\frac{1}{2}
\end{array}\right] ; \quad y_{3}^{(1)}=\left[\begin{array}{c}
0 \\
0 \\
0 \\
-1 \\
0
\end{array}\right] \text {, } \\
& y_{1}^{(2)}=\left[\begin{array}{c}
a_{1} \\
b_{1} \\
0 \\
0 \\
0
\end{array}\right] ; \quad y_{2}^{(2)}=\left[\begin{array}{c}
a_{2} \\
0 \\
c_{2} \\
0 \\
-\frac{1}{4}
\end{array}\right] ; \quad y_{3}^{(2)}=\left[\begin{array}{c}
0 \\
b_{3} \\
c_{3} \\
-1 \\
0
\end{array}\right] \text {. }
\end{aligned}
$$

With $y_{i}^{(1)}(i=1,2,3)$ now fully determined, the Dalgarno-Stewart identities yield

$$
\begin{aligned}
& \lambda_{1}^{(3)}=\left\langle y_{1}^{(1)},\left(A_{1}-\lambda^{(1)} I\right) y_{1}^{(1)}\right\rangle=0, \\
& \lambda_{2}^{(3)}=\left\langle y_{2}^{(1)},\left(A_{1}-\lambda^{(1)} I\right) y_{2}^{(1)}\right\rangle=-\frac{1}{4}, \\
& \lambda_{3}^{(3)}=\left\langle y_{3}^{(1)},\left(A_{1}-\lambda^{(1)} I\right) y_{3}^{(1)}\right\rangle=-1 .
\end{aligned}
$$

Solving (2.5), for $k=3$,

$$
\begin{aligned}
\left(A_{0}-\lambda^{(0)} I\right) y_{i}^{(3)}= & -\left(A_{1}-\lambda^{(1)} I\right) y_{i}^{(2)} \\
& +\lambda_{i}^{(2)} y_{i}^{(1)}+\lambda_{i}^{(3)} y_{i}^{(0)} \quad(i=1,2,3),
\end{aligned}
$$


produces

$$
y_{1}^{(3)}=\left[\begin{array}{c}
u_{1} \\
v_{1} \\
0 \\
-a_{1} \\
-\frac{b_{1}}{2}
\end{array}\right], \quad y_{2}^{(3)}=\left[\begin{array}{c}
u_{2} \\
0 \\
w_{2} \\
-a_{2} \\
0
\end{array}\right], \quad y_{3}^{(3)}=\left[\begin{array}{c}
0 \\
v_{3} \\
w_{3} \\
0 \\
-\frac{b_{3}}{2}
\end{array}\right]
$$

where we have invoked intermediate normalization. As before, $y_{i}^{(3)}(i=$ $1,2,3)$ are not yet fully determined.

We now enforce solvability of (2.5) for $k=4$ :

$$
\left\langle y_{j}^{(0)},-\left(A_{1}-\lambda^{(1)} I\right) y_{i}^{(3)}+\lambda_{i}^{(2)} y_{i}^{(2)}+\lambda_{i}^{(3)} y_{i}^{(1)}+\lambda_{i}^{(4)} y_{i}^{(0)}\right\rangle=0 \quad(i \neq j),
$$

thereby fully determining

$$
y_{1}^{(2)}=\left[\begin{array}{l}
0 \\
0 \\
0 \\
0 \\
0
\end{array}\right], \quad y_{2}^{(2)}=\left[\begin{array}{c}
0 \\
0 \\
0 \\
0 \\
-\frac{1}{4}
\end{array}\right], \quad y_{3}^{(2)}=\left[\begin{array}{c}
0 \\
0 \\
0 \\
-1 \\
0
\end{array}\right]
$$

Subsequent application of the Dalgarno-Stewart identities yields

$$
\begin{aligned}
& \lambda_{1}^{(4)}=\left\langle y_{1}^{(1)},\left(A_{1}-\lambda^{(1)} I\right) y_{1}^{(2)}\right\rangle-\lambda_{1}^{(2)}\left\langle y_{1}^{(1)}, y_{1}^{(1)}\right\rangle=0, \\
& \lambda_{1}^{(5)}=\left\langle y_{1}^{(2)},\left(A_{1}-\lambda^{(1)} I\right) y_{1}^{(2)}\right\rangle-2 \lambda_{1}^{(2)}\left\langle y_{1}^{(2)}, y_{1}^{(1)}\right\rangle-\lambda_{1}^{(3)}\left\langle y_{1}^{(1)}, y_{1}^{(1)}\right\rangle=0, \\
& \lambda_{2}^{(4)}=\left\langle y_{2}^{(1)},\left(A_{1}-\lambda^{(1)} I\right) y_{2}^{(2)}\right\rangle-\lambda_{2}^{(2)}\left\langle y_{2}^{(1)}, y_{2}^{(1)}\right\rangle=0, \\
& \lambda_{2}^{(5)}=\left\langle y_{2}^{(2)},\left(A_{1}-\lambda^{(1)} I\right) y_{2}^{(2)}\right\rangle-2 \lambda_{2}^{(2)}\left\langle y_{2}^{(2)}, y_{2}^{(1)}\right\rangle-\lambda_{2}^{(3)}\left\langle y_{2}^{(1)}, y_{2}^{(1)}\right\rangle=\frac{1}{8}, \\
& \lambda_{3}^{(4)}=\left\langle y_{3}^{(1)},\left(A_{1}-\lambda^{(1)} I\right) y_{3}^{(2)}\right\rangle-\lambda_{3}^{(2)}\left\langle y_{3}^{(1)}, y_{3}^{(1)}\right\rangle=0, \\
& \lambda_{3}^{(5)}=\left\langle y_{3}^{(2)},\left(A_{1}-\lambda^{(1)} I\right) y_{3}^{(2)}\right\rangle-2 \lambda_{3}^{(2)}\left\langle y_{3}^{(2)}, y_{3}^{(1)}\right\rangle-\lambda_{3}^{(3)}\left\langle y_{3}^{(1)}, y_{3}^{(1)}\right\rangle=2 .
\end{aligned}
$$

\subsection{Nth order degeneracy}

We now consider the case of $N$ th order degeneracy which is characterized by the conditions $\lambda_{1}^{(j)}=\lambda_{2}^{(j)}=\cdots=\lambda_{m}^{(j)}=\lambda^{(j)}(j=0, \ldots, N-1)$, while $\lambda_{i}^{(N)}(i=1, \ldots, m)$ are all distinct. Thus, even though $\lambda^{(j)}(j=0, \ldots, N-1)$ 
are determinate, $\left\{y_{i}^{(0)}\right\}_{i=1}^{m}$ are still indeterminate after enforcing solvability of (2.5) for $k=N-1$.

Hence, we will determine $\left\{\lambda_{i}^{(N)} ; y_{i}^{(0)}\right\}_{i=1}^{m}$ by insisting that $(2.5)$ be solvable for $k=N$ and $i=1, \ldots, m$. This requirement is equivalent to the condition that, for each fixed $i$,

$$
\left\langle x_{\mu}^{(0)},-\left(A_{1}-\lambda^{(1)} I\right) y_{i}^{(N-1)}+\lambda^{(2)} y_{i}^{(N-2)}+\cdots+\lambda_{i}^{(N)} y_{i}^{(0)}\right\rangle=0 \quad(\mu=1, \ldots, m) .
$$

Inserting (3.1) as well as (3.7) with $k=1, \ldots, N-1$ and invoking the orthonormality of $\left\{x_{\mu}^{(0)}\right\}_{\mu=1}^{m}$, we arrive at, in matrix form,

$$
\left[\begin{array}{ccc}
\left\langle x_{1}^{(0)}, M^{(N)} x_{1}^{(0)}\right\rangle & \cdots & \left\langle x_{1}^{(0)}, M^{(N)} x_{m}^{(0)}\right\rangle \\
\vdots & \ddots & \vdots \\
\left\langle x_{m}^{(0)}, M^{(N)} x_{1}^{(0)}\right\rangle & \cdots & \left\langle x_{m}^{(0)}, M^{(N)} x_{m}^{(0)}\right\rangle
\end{array}\right]\left[\begin{array}{c}
a_{1}^{(i)} \\
\vdots \\
a_{m}^{(i)}
\end{array}\right]=\lambda_{i}^{(N)}\left[\begin{array}{c}
a_{1}^{(i)} \\
\vdots \\
a_{m}^{(i)}
\end{array}\right]
$$

where $M^{(N)}$ is specified by the recurrence relation

$$
\begin{aligned}
M^{(1)}= & A_{1} \\
M^{(2)}= & \left(\lambda^{(1)} I-M^{(1)}\right)\left(A_{0}-\lambda^{(0)} I\right)^{\dagger}\left(A_{1}-\lambda^{(1)} I\right) \\
M^{(3)}= & \left(\lambda^{(2)} I-M^{(2)}\right)\left(A_{0}-\lambda^{(0)} I\right)^{\dagger}\left(A_{1}-\lambda^{(1)} I\right) \\
& +\lambda^{(2)}\left(A_{1}-\lambda^{(1)} I\right)\left(A_{0}-\lambda^{(0)} I\right)^{\dagger} \\
M^{(N)}= & \left(\lambda^{(N-1)} I-M^{(N-1)}\right)\left(A_{0}-\lambda^{(0)} I\right)^{\dagger}\left(A_{1}-\lambda^{(1)} I\right) \\
& -\sum_{l=2}^{N-3} \lambda^{(l)}\left(\lambda^{(N-l)} I-M^{(N-l)}\right)\left(A_{0}-\lambda^{(0)} I\right)^{\dagger} \\
& -\lambda^{(N-2)}\left[\left(A_{1}-\lambda^{(1)} I\right)\left(A_{0}-\lambda^{(0)} I\right)^{\dagger}\left(A_{1}-\lambda^{(1)} I\right)+\lambda^{(2)} I\right]\left(A_{0}-\lambda^{(0)} I\right)^{\dagger} \\
& +\lambda^{(N-1)}\left(A_{1}-\lambda^{(1)} I\right)\left(A_{0}-\lambda^{(0)} I\right)^{\dagger} \quad(N=4,5, \ldots) .
\end{aligned}
$$

Thus, each $\lambda_{i}^{(N)}$ is an eigenvalue with corresponding eigenvector $\left[a_{1}^{(i)}, \ldots, a_{m}^{(i)}\right]^{T}$ of the matrix $M$ defined by $M_{\mu, v}=\left\langle x_{\mu}^{(0)}, M^{(N)} x_{v}^{(0)}\right\rangle(\mu, v=$ $1, \ldots, m)$. It is important to note that, while this recurrence relation guarantees that $\left\{\lambda_{i}^{(N)} ; y_{i}^{(0)}\right\}_{i=1}^{m}$ are well defined by enforcing solvability of (2.5) for $k=N, M^{(N)}$ need not be explicitly computed.

By assumption, the symmetric matrix $M$ has $m$ distinct real eigenvalues and hence orthonormal eigenvectors described by (3.2). These, in turn, may be used in concert with (3.1) to yield the desired special unperturbed eigenvectors alluded to above. 
Now that $\left\{y_{i}^{(0)}\right\}_{i=1}^{m}$ are fully determined, we have by the combination of (3.2) and (3.47) the identities

$$
\left\langle y_{i}^{(0)}, M^{(N)} y_{j}^{(0)}\right\rangle=\lambda_{i}^{(N)} \cdot \delta_{i, j}
$$

The remaining eigenvalue corrections $\lambda_{i}^{(k)}(k \geq N+1)$ may be obtained from the Dalgarno-Stewart identities.

Analogous to the cases of first-order and second-order degeneracies, $\beta_{j, k}^{(i)}(i \neq j)$ of (3.7) are to be determined from the condition that (2.5) be solvable for $k \leftarrow k+N$ and $i=1, \ldots, m$. Since, by design, (2.5) is solvable for $k=1, \ldots, N$, we may proceed recursively. After considerable algebraic manipulation, the end result is

$$
\beta_{j, k}^{(i)}=\frac{\left\langle y_{j}^{(0)}, M^{(N)} \hat{y}_{i}^{(k)}\right\rangle-\sum_{l=1}^{k-1} \lambda_{i}^{(k-l+N)} \beta_{j, l}^{(i)}}{\lambda_{i}^{(N)}-\lambda_{j}^{(N)}} \quad(i \neq j) .
$$

The existence of this formula guarantees that each $y_{i}^{(k)}$ is uniquely determined by enforcing solvability of (2.5) for $k \leftarrow k+N$.

Example 3.3. Define

$$
A_{0}=\left[\begin{array}{llll}
1 & 0 & 0 & 0 \\
0 & 1 & 0 & 0 \\
0 & 0 & 2 & 0 \\
0 & 0 & 0 & 2
\end{array}\right], \quad A_{1}=\left[\begin{array}{rrrr}
1 & 0 & 1 & 1 \\
0 & 1 & 1 & -1 \\
1 & 1 & 1 & 0 \\
1 & -1 & 0 & 0
\end{array}\right]
$$

Using Matlab's Symbolic Toolbox, we find that

$$
\begin{aligned}
& \lambda_{1}(\epsilon)=1+\epsilon-2 \epsilon^{2}+4 \epsilon^{4}+0 \cdot \epsilon^{5}+\cdots \\
& \lambda_{2}(\epsilon)=1+\epsilon-2 \epsilon^{2}-2 \epsilon^{3}+2 \epsilon^{4}+10 \epsilon^{5}+\cdots \\
& \lambda_{3}(\epsilon)=2+2 \epsilon^{2}+2 \epsilon^{3}-2 \epsilon^{4}-10 \epsilon^{5}+\cdots \\
& \lambda_{4}(\epsilon)=2+\epsilon+2 \epsilon^{2}-4 \epsilon^{4}+0 \cdot \epsilon^{5}+\cdots
\end{aligned}
$$

We focus on the third-order degeneracy amongst $\lambda_{1}^{(0)}=\lambda_{2}^{(0)}=\lambda^{(0)}=1$. With the choice

$$
x_{1}^{(0)}=\left[\begin{array}{l}
1 \\
0 \\
0 \\
0
\end{array}\right], \quad x_{2}^{(0)}=\left[\begin{array}{l}
0 \\
1 \\
0 \\
0
\end{array}\right]
$$


476 Pseudoinverse formulation of perturbation theory

we have from (3.4), which enforces solvability of (2.5) for $k=1$,

$$
M=\left[\begin{array}{ll}
1 & 0 \\
0 & 1
\end{array}\right]
$$

with double eigenvalue $\lambda^{(1)}=1$. Equation (3.24), which enforces solvability of (2.5) for $k=2$, yields

$$
M=\left[\begin{array}{cc}
-2 & 0 \\
0 & -2
\end{array}\right]
$$

with double eigenvalue $\lambda^{(2)}=-2$.

Moving on to (3.47) with $N=3$, which enforces solvability of (2.5) for $k=3$, we have

$$
M=\left[\begin{array}{cc}
-1 & 1 \\
1 & -1
\end{array}\right]
$$

with eigenpairs

$$
\lambda_{1}^{(3)}=0, \quad\left[\begin{array}{l}
a_{1}^{(1)} \\
a_{2}^{(1)}
\end{array}\right]=\left[\begin{array}{c}
\frac{1}{\sqrt{2}} \\
\frac{1}{\sqrt{2}}
\end{array}\right] ; \quad \lambda_{2}^{(3)}=-2, \quad\left[\begin{array}{l}
a_{1}^{(2)} \\
a_{2}^{(2)}
\end{array}\right]=\left[\begin{array}{c}
\frac{1}{\sqrt{2}} \\
-\frac{1}{\sqrt{2}}
\end{array}\right] \text {. }
$$

Availing ourselves of (3.1), the special unperturbed eigenvectors are now fully determined as

$$
y_{1}^{(0)}=\left[\begin{array}{c}
\frac{1}{\sqrt{2}} \\
\frac{1}{\sqrt{2}} \\
0 \\
0
\end{array}\right], \quad y_{2}^{(0)}=\left[\begin{array}{c}
\frac{1}{\sqrt{2}} \\
-\frac{1}{\sqrt{2}} \\
0 \\
0
\end{array}\right] \text {. }
$$

Solving (2.5), for $k=1$,

$$
\left(A_{0}-\lambda^{(0)} I\right) y_{i}^{(1)}=-\left(A_{1}-\lambda^{(1)} I\right) y_{i}^{(0)} \quad(i=1,2),
$$


produces

$$
y_{1}^{(1)}=\left[\begin{array}{c}
a \\
-a \\
-\sqrt{2} \\
0
\end{array}\right], \quad y_{2}^{(1)}=\left[\begin{array}{c}
b \\
b \\
0 \\
-\sqrt{2}
\end{array}\right]
$$

where we have invoked intermediate normalization. Observe that $y_{i}^{(1)}$ $(i=1,2)$ are not yet fully determined.

Solving (2.5), for $k=2$,

$$
\left(A_{0}-\lambda^{(0)} I\right) y_{i}^{(2)}=-\left(A_{1}-\lambda^{(1)} I\right) y_{i}^{(1)}+\lambda^{(2)} y_{i}^{(0)} \quad(i=1,2),
$$

produces

$$
y_{1}^{(2)}=\left[\begin{array}{c}
c \\
-c \\
0 \\
-2 a
\end{array}\right], \quad y_{2}^{(2)}=\left[\begin{array}{c}
d \\
d \\
-2 b \\
-\sqrt{2}
\end{array}\right] \text {, }
$$

where we have invoked intermediate normalization. Likewise, $y_{i}^{(2)}(i=$ $1,2)$ are not yet fully determined.

Solving (2.5), for $k=3$,

$$
\left(A_{0}-\lambda^{(0)} I\right) y_{i}^{(3)}=-\left(A_{1}-\lambda^{(1)} I\right) y_{i}^{(2)}+\lambda^{(2)} y_{i}^{(1)}+\lambda_{i}^{(3)} y_{i}^{(0)} \quad(i=1,2),
$$

produces

$$
y_{1}^{(3)}=\left[\begin{array}{c}
e \\
-e \\
2 \sqrt{2} \\
-2 c-2 a
\end{array}\right], \quad y_{2}^{(3)}=\left[\begin{array}{c}
f \\
f \\
-2 d \\
\sqrt{2}
\end{array}\right]
$$

where we have invoked intermediate normalization. Likewise, $y_{i}^{(3)}(i=$ $1,2)$ are not yet fully determined.

We next enforce solvability of (2.5) for $k=4$ :

$$
\left\langle y_{j}^{(0)},-\left(A_{1}-\lambda^{(1)} I\right) y_{i}^{(3)}+\lambda^{(2)} y_{i}^{(2)}+\lambda_{i}^{(3)} y_{i}^{(1)}+\lambda_{i}^{(4)} y_{i}^{(0)}\right\rangle=0 \quad(i \neq j),
$$


478 Pseudoinverse formulation of perturbation theory

thereby producing

$$
\begin{array}{ll}
y_{1}^{(1)}=\left[\begin{array}{c}
0 \\
0 \\
-\sqrt{2} \\
0
\end{array}\right] ; & y_{2}^{(1)}=\left[\begin{array}{c}
0 \\
0 \\
0 \\
-\sqrt{2}
\end{array}\right], \\
y_{1}^{(2)}=\left[\begin{array}{c}
c \\
-c \\
0 \\
0
\end{array}\right] ; & y_{2}^{(2)}=\left[\begin{array}{c}
d \\
d \\
0 \\
-\sqrt{2}
\end{array}\right], \\
y_{1}^{(3)}=\left[\begin{array}{c}
e \\
-e \\
2 \sqrt{2} \\
-2 c
\end{array}\right] ; & y_{2}^{(3)}=\left[\begin{array}{c}
f \\
f \\
-2 d \\
\sqrt{2}
\end{array}\right] .
\end{array}
$$

Observe that $y_{i}^{(1)}(i=1,2)$ are now fully determined, while $y_{i}^{(2)}(i=1,2)$ and $y_{i}^{(3)}(i=1,2)$ are not yet completely specified.

Solving (2.5), for $k=4$,

$$
\begin{aligned}
\left(A_{0}-\lambda^{(0)} I\right) y_{i}^{(4)}= & -\left(A_{1}-\lambda^{(1)} I\right) y_{i}^{(3)}+\lambda^{(2)} y_{i}^{(2)} \\
& +\lambda_{i}^{(3)} y_{i}^{(1)}+\lambda_{i}^{(4)} y_{i}^{(0)} \quad(i=1,2),
\end{aligned}
$$

produces

$$
y_{1}^{(4)}=\left[\begin{array}{c}
g \\
h \\
0 \\
-2 e-2 c
\end{array}\right], \quad y_{2}^{(4)}=\left[\begin{array}{c}
u \\
v \\
-2 f \\
5 \sqrt{2}
\end{array}\right]
$$

where we have invoked intermediate normalization. As before, $y_{i}^{(4)}(i=$ $1,2)$ are not yet fully determined.

We now enforce solvability of (2.5) for $k=5$ :

$$
\left\langle y_{j}^{(0)},-\left(A_{1}-\lambda^{(1)} I\right) y_{i}^{(4)}+\lambda^{(2)} y_{i}^{(3)}+\lambda_{i}^{(3)} y_{i}^{(2)}+\lambda_{i}^{(4)} y_{i}^{(1)}+\lambda_{i}^{(5)} y_{i}^{(0)}\right\rangle=0 \quad(i \neq j),
$$


thereby fully determining

$$
y_{1}^{(2)}=\left[\begin{array}{l}
0 \\
0 \\
0 \\
0
\end{array}\right], \quad y_{2}^{(2)}=\left[\begin{array}{c}
0 \\
0 \\
0 \\
-\sqrt{2}
\end{array}\right]
$$

and further specifying

$$
\begin{array}{ll}
y_{1}^{(3)}=\left[\begin{array}{c}
e \\
-e \\
2 \sqrt{2} \\
0
\end{array}\right], & y_{2}^{(3)}=\left[\begin{array}{c}
f \\
f \\
0 \\
\sqrt{2}
\end{array}\right], \\
y_{1}^{(4)}=\left[\begin{array}{c}
g \\
h \\
0 \\
-2 e
\end{array}\right], & y_{2}^{(4)}=\left[\begin{array}{c}
u \\
v \\
-2 f \\
5 \sqrt{2}
\end{array}\right] .
\end{array}
$$

Subsequent application of the Dalgarno-Stewart identities yields

$$
\begin{aligned}
& \lambda_{1}^{(4)}=\left\langle y_{1}^{(1)},\left(A_{1}-\lambda^{(1)} I\right) y_{1}^{(2)}\right\rangle-\lambda_{1}^{(2)}\left\langle y_{1}^{(1)}, y_{1}^{(1)}\right\rangle=4, \\
& \lambda_{1}^{(5)}=\left\langle y_{1}^{(2)},\left(A_{1}-\lambda^{(1)} I\right) y_{1}^{(2)}\right\rangle-2 \lambda_{1}^{(2)}\left\langle y_{1}^{(2)}, y_{1}^{(1)}\right\rangle-\lambda_{1}^{(3)}\left\langle y_{1}^{(1)}, y_{1}^{(1)}\right\rangle=0, \\
& \lambda_{2}^{(4)}=\left\langle y_{2}^{(1)},\left(A_{1}-\lambda^{(1)} I\right) y_{2}^{(2)}\right\rangle-\lambda_{2}^{(2)}\left\langle y_{2}^{(1)}, y_{2}^{(1)}\right\rangle=2, \\
& \lambda_{2}^{(5)}=\left\langle y_{2}^{(2)},\left(A_{1}-\lambda^{(1)} I\right) y_{2}^{(2)}\right\rangle-2 \lambda_{2}^{(2)}\left\langle y_{2}^{(2)}, y_{2}^{(1)}\right\rangle-\lambda_{2}^{(3)}\left\langle y_{2}^{(1)}, y_{2}^{(1)}\right\rangle=10 .
\end{aligned}
$$

\subsection{Mixed degeneracy}

Finally, we arrive at the most general case of mixed degeneracy wherein a degeneracy (multiple eigenvalue) is partially resolved at more than a single order. The analysis expounded upon in the previous sections comprises the core of the procedure for the complete resolution of mixed degeneracy. The following modifications suffice.

During the Rayleigh-Schrödinger procedure, whenever an eigenvalue branches by reduction in multiplicity at any order, one simply replaces the $x_{\mu}$ of (3.47) by any convenient orthonormal basis $z_{\mu}$ for the reduced eigenspace. Of course, this new basis is composed of some a priori unknown linear combination of the original basis. Equation (3.50) will still be valid where $N$ is the order of correction where the degeneracy between $\lambda_{i}$ and $\lambda_{j}$ is resolved. Thus, in general, if $\lambda_{i}$ is degenerate to $N$ th order, then $y_{i}^{(k)}$ will be fully determined by enforcing the solvability of (2.5) with $k \leftarrow k+N$. 
We now present a final example which illustrates this general procedure. This example features a triple eigenvalue which branches into a single first-order degenerate eigenvalue, together with a pair of secondorder degenerate eigenvalues. Hence, we observe features of both Examples 3.1 and 3.2 appearing in tandem.

Example 3.4. Define

$$
A_{0}=\left[\begin{array}{llll}
0 & 0 & 0 & 0 \\
0 & 0 & 0 & 0 \\
0 & 0 & 0 & 0 \\
0 & 0 & 0 & 1
\end{array}\right], \quad A_{1}=\left[\begin{array}{llll}
1 & 0 & 0 & 1 \\
0 & 1 & 0 & 0 \\
0 & 0 & 0 & 0 \\
1 & 0 & 0 & 0
\end{array}\right]
$$

Using Matlab's Symbolic Toolbox, we find that

$$
\begin{aligned}
& \lambda_{1}(\epsilon)=\epsilon \\
& \lambda_{2}(\epsilon)=\epsilon-\epsilon^{2}-\epsilon^{3}+2 \epsilon^{5}+\cdots, \\
& \lambda_{3}(\epsilon)=0 \\
& \lambda_{4}(\epsilon)=1+\epsilon^{2}+\epsilon^{3}-2 \epsilon^{5}+\cdots .
\end{aligned}
$$

We focus on the mixed degeneracy amongst $\lambda_{1}^{(0)}=\lambda_{2}^{(0)}=\lambda_{3}^{(0)}=\lambda^{(0)}=0$. With the choice

$$
x_{1}^{(0)}=\left[\begin{array}{l}
1 \\
0 \\
0 \\
0
\end{array}\right], \quad x_{2}^{(0)}=\left[\begin{array}{l}
0 \\
1 \\
0 \\
0
\end{array}\right], \quad x_{3}^{(0)}=\left[\begin{array}{l}
0 \\
0 \\
1 \\
0
\end{array}\right],
$$

we have from (3.4), which enforces solvability of (2.5) for $k=1$,

$$
M=\left[\begin{array}{lll}
1 & 0 & 0 \\
0 & 1 & 0 \\
0 & 0 & 0
\end{array}\right]
$$

with eigenvalues $\lambda_{1}^{(1)}=\lambda_{2}^{(1)}=\lambda^{(1)}=1, \lambda_{3}^{(1)}=0$.

Thus, $y_{1}^{(0)}$ and $y_{2}^{(0)}$ are indeterminate, while

$$
\left[\begin{array}{l}
a_{1}^{(3)} \\
a_{2}^{(3)} \\
a_{3}^{(3)}
\end{array}\right]=\left[\begin{array}{l}
0 \\
0 \\
1
\end{array}\right] \Longrightarrow y_{3}^{(0)}=\left[\begin{array}{l}
0 \\
0 \\
1 \\
0
\end{array}\right]
$$


Introducing the new basis

$$
z_{1}^{(0)}=\left[\begin{array}{c}
\frac{1}{\sqrt{2}} \\
\frac{1}{\sqrt{2}} \\
0 \\
0
\end{array}\right], \quad z_{2}^{(0)}=\left[\begin{array}{c}
\frac{1}{\sqrt{2}} \\
-\frac{1}{\sqrt{2}} \\
0 \\
0
\end{array}\right]
$$

we now seek $y_{1}^{(0)}$ and $y_{2}^{(0)}$ in the form

$$
y_{1}^{(0)}=b_{1}^{(1)} z_{1}^{(0)}+b_{2}^{(1)} z_{2}^{(0)}, \quad y_{2}^{(0)}=b_{1}^{(2)} z_{1}^{(0)}+b_{2}^{(2)} z_{2}^{(0)}
$$

with orthonormal $\left\{\left[b_{1}^{(1)}, b_{2}^{(1)}\right]^{T},\left[b_{1}^{(2)}, b_{2}^{(2)}\right]^{T}\right\}$.

Solving (2.5), for $k=1$,

$$
\left(A_{0}-\lambda^{(0)} I\right) y_{i}^{(1)}=-\left(A_{1}-\lambda_{i}^{(1)} I\right) y_{i}^{(0)} \quad(i=1,2,3),
$$

produces

$$
\begin{aligned}
& y_{1}^{(1)}=\left[\begin{array}{c}
\alpha_{1} \\
\beta_{1} \\
\gamma_{1} \\
\left(b_{1}^{(1)}+b_{2}^{(1)}\right) \\
-\sqrt{2}
\end{array}\right], \\
& y_{2}^{(1)}=\left[\begin{array}{c}
\alpha_{2} \\
\beta_{2} \\
\gamma_{2} \\
-\frac{\left(b_{1}^{(2)}+b_{2}^{(2)}\right)}{\sqrt{2}}
\end{array}\right], \\
& y_{3}^{(1)}=\left[\begin{array}{c}
\alpha_{3} \\
\beta_{3} \\
\gamma_{3} \\
0
\end{array}\right] .
\end{aligned}
$$

Now, enforcing solvability of (2.5), for $k=2$,

$$
-\left(A_{1}-\lambda_{i}^{(1)} I\right) y_{i}^{(1)}+\lambda_{i}^{(2)} y_{i}^{(0)} \perp\left\{z_{1}^{(0)}, z_{2}^{(0)}, y_{3}^{(0)}\right\} \quad(i=1,2,3),
$$


482 Pseudoinverse formulation of perturbation theory

we arrive at

$$
M=\left[\begin{array}{rr}
-\frac{1}{2} & -\frac{1}{2} \\
-\frac{1}{2} & -\frac{1}{2}
\end{array}\right]
$$

with eigenpairs

$$
\begin{aligned}
& \lambda_{1}^{(2)}=0, \quad\left[\begin{array}{l}
b_{1}^{(1)} \\
b_{2}^{(1)}
\end{array}\right]=\left[\begin{array}{c}
\frac{1}{\sqrt{2}} \\
-\frac{1}{\sqrt{2}}
\end{array}\right] ; \\
& \lambda_{2}^{(2)}=-1, \quad\left[\begin{array}{l}
b_{1}^{(2)} \\
b_{2}^{(2)}
\end{array}\right]=\left[\begin{array}{c}
\frac{1}{\sqrt{2}} \\
\frac{1}{\sqrt{2}}
\end{array}\right] \Longrightarrow y_{1}^{(0)}=\left[\begin{array}{l}
0 \\
1 \\
0 \\
0
\end{array}\right] ; \quad y_{2}^{(0)}=\left[\begin{array}{l}
1 \\
0 \\
0 \\
0
\end{array}\right], \\
& y_{1}^{(1)}=\left[\begin{array}{c}
\alpha_{1} \\
\beta_{1} \\
0 \\
0
\end{array}\right] ; \quad y_{2}^{(1)}=\left[\begin{array}{c}
\alpha_{2} \\
\beta_{2} \\
0 \\
-1
\end{array}\right] ; \quad y_{3}^{(1)}=\left[\begin{array}{l}
0 \\
0 \\
0 \\
0
\end{array}\right],
\end{aligned}
$$

as well as $\lambda_{3}^{(2)}=0$, where we have invoked intermediate normalization. Observe that $y_{1}^{(1)}$ and $y_{2}^{(1)}$ have not yet been fully determined, while $y_{3}^{(1)}$ has indeed been completely specified.

Solving (2.5), for $k=2$,

$$
\left(A_{0}-\lambda^{(0)} I\right) y_{i}^{(2)}=-\left(A_{1}-\lambda_{i}^{(1)} I\right) y_{i}^{(1)}+\lambda_{i}^{(2)} y_{i}^{(0)} \quad(i=1,2,3),
$$

produces

$$
y_{1}^{(2)}=\left[\begin{array}{c}
a_{1} \\
0 \\
c_{1} \\
-\alpha_{1}
\end{array}\right], \quad y_{2}^{(2)}=\left[\begin{array}{c}
0 \\
b_{2} \\
c_{2} \\
-1
\end{array}\right], \quad y_{3}^{(2)}=\left[\begin{array}{c}
a_{3} \\
b_{3} \\
0 \\
0
\end{array}\right]
$$

where we have invoked intermediate normalization.

We next enforce solvability of (2.5) for $k=3$ :

$$
\left\langle y_{j}^{(0)},-\left(A_{1}-\lambda_{i}^{(1)} I\right) y_{i}^{(2)}+\lambda_{i}^{(2)} y_{i}^{(1)}+\lambda_{i}^{(3)} y_{i}^{(0)}\right\rangle=0 \quad(i \neq j),
$$


thereby producing

$$
\begin{array}{ll}
y_{1}^{(1)}=\left[\begin{array}{l}
0 \\
0 \\
0 \\
0
\end{array}\right] ; & y_{2}^{(1)}=\left[\begin{array}{c}
0 \\
0 \\
0 \\
-1
\end{array}\right], \\
y_{1}^{(2)}=\left[\begin{array}{l}
a_{1} \\
0 \\
0 \\
0
\end{array}\right] ; \quad y_{2}^{(2)}=\left[\begin{array}{c}
0 \\
b_{2} \\
0 \\
-1
\end{array}\right] ; \quad y_{3}^{(2)}=\left[\begin{array}{l}
0 \\
0 \\
0 \\
0
\end{array}\right] .
\end{array}
$$

With $y_{i}^{(1)}(i=1,2,3)$ now fully determined, the Dalgarno-Stewart identities yield

$$
\begin{aligned}
& \lambda_{1}^{(3)}=\left\langle y_{1}^{(1)},\left(A_{1}-\lambda^{(1)} I\right) y_{1}^{(1)}\right\rangle=0, \\
& \lambda_{2}^{(3)}=\left\langle y_{2}^{(1)},\left(A_{1}-\lambda^{(1)} I\right) y_{2}^{(1)}\right\rangle=-1, \\
& \lambda_{3}^{(3)}=\left\langle y_{3}^{(1)},\left(A_{1}-\lambda_{3}^{(1)} I\right) y_{3}^{(1)}\right\rangle=0 .
\end{aligned}
$$

Solving (2.5), for $k=3$,

$$
\left(A_{0}-\lambda^{(0)} I\right) y_{i}^{(3)}=-\left(A_{1}-\lambda^{(1)} I\right) y_{i}^{(2)}+\lambda_{i}^{(2)} y_{i}^{(1)}+\lambda_{i}^{(3)} y_{i}^{(0)} \quad(i=1,2),
$$

produces

$$
y_{1}^{(3)}=\left[\begin{array}{c}
u_{1} \\
0 \\
w_{1} \\
-a_{1}
\end{array}\right], \quad y_{2}^{(3)}=\left[\begin{array}{c}
0 \\
v_{2} \\
w_{2} \\
0
\end{array}\right]
$$

where we have invoked intermediate normalization.

We now enforce solvability of (2.5) for $k=4$ :

$$
\left\langle y_{j}^{(0)},-\left(A_{1}-\lambda^{(1)} I\right) y_{i}^{(3)}+\lambda_{i}^{(2)} y_{i}^{(2)}+\lambda_{i}^{(3)} y_{i}^{(1)}+\lambda_{i}^{(4)} y_{i}^{(0)}\right\rangle=0 \quad(i \neq j),
$$


thereby fully determining

$$
y_{1}^{(2)}=\left[\begin{array}{l}
0 \\
0 \\
0 \\
0
\end{array}\right], \quad y_{2}^{(2)}=\left[\begin{array}{c}
0 \\
0 \\
0 \\
-1
\end{array}\right]
$$

Subsequent application of the Dalgarno-Stewart identities yields

$$
\begin{aligned}
& \lambda_{1}^{(4)}=\left\langle y_{1}^{(1)},\left(A_{1}-\lambda^{(1)} I\right) y_{1}^{(2)}\right\rangle-\lambda_{1}^{(2)}\left\langle y_{1}^{(1)}, y_{1}^{(1)}\right\rangle=0, \\
& \lambda_{1}^{(5)}=\left\langle y_{1}^{(2)},\left(A_{1}-\lambda^{(1)} I\right) y_{1}^{(2)}\right\rangle-2 \lambda_{1}^{(2)}\left\langle y_{1}^{(2)}, y_{1}^{(1)}\right\rangle-\lambda_{1}^{(3)}\left\langle y_{1}^{(1)}, y_{1}^{(1)}\right\rangle=0, \\
& \lambda_{2}^{(4)}=\left\langle y_{2}^{(1)},\left(A_{1}-\lambda^{(1)} I\right) y_{2}^{(2)}\right\rangle-\lambda_{2}^{(2)}\left\langle y_{2}^{(1)}, y_{2}^{(1)}\right\rangle=0, \\
& \lambda_{2}^{(5)}=\left\langle y_{2}^{(2)},\left(A_{1}-\lambda^{(1)} I\right) y_{2}^{(2)}\right\rangle-2 \lambda_{2}^{(2)}\left\langle y_{2}^{(2)}, y_{2}^{(1)}\right\rangle-\lambda_{2}^{(3)}\left\langle y_{2}^{(1)}, y_{2}^{(1)}\right\rangle=2, \\
& \lambda_{3}^{(4)}=\left\langle y_{3}^{(1)},\left(A_{1}-\lambda_{3}^{(1)} I\right) y_{3}^{(2)}\right\rangle-\lambda_{3}^{(2)}\left\langle y_{3}^{(1)}, y_{3}^{(1)}\right\rangle=0, \\
& \lambda_{3}^{(5)}=\left\langle y_{3}^{(2)},\left(A_{1}-\lambda_{3}^{(1)} I\right) y_{3}^{(2)}\right\rangle-2 \lambda_{3}^{(2)}\left\langle y_{3}^{(2)}, y_{3}^{(1)}\right\rangle-\lambda_{3}^{(3)}\left\langle y_{3}^{(1)}, y_{3}^{(1)}\right\rangle=0 .
\end{aligned}
$$

\section{Conclusion}

In this paper, we have endeavored to provide a comprehensive and unified account of the Rayleigh-Schrödinger perturbation theory for the symmetric matrix eigenvalue problem. The cornerstone of our development has been the Moore-Penrose pseudoinverse. Not only does this approach permit a direct analysis of the properties of this procedure but it also obviates the need of alternative approaches for the computation of all of the eigenvectors of the unperturbed matrix. Instead, we only require the unperturbed eigenvectors corresponding to those eigenvalues of interest.

The focal point of this investigation has been the degenerate case. In the light of the inherent complexity of this topic, we have built up the theory gradually with the expectation that the reader would thence not be swept away in a torrent of formulae. At each stage, we have attempted to make the subject more accessible by a judicious choice of an illustrative example. (Observe that all of the examples were worked through without explicit computation of the pseudoinverse.) Hopefully, these efforts have met with a modicum of success. 


\section{References}

[1] F. W. Byron Jr. and R. W. Fuller, Mathematics of Classical and Quantum Physics, Dover Publications, New York, 1992.

[2] A. Dalgarno and A. L. Stewart, On the perturbation theory of small disturbances, Proc. Roy. Soc. London Ser. A 238 (1956), 269-275.

[3] K. O. Friedrichs, Perturbation of Spectra in Hilbert Space, Lectures in Applied Mathematics, vol. 3, American Mathematical Society, Rhode Island, 1965.

[4] J. O. Hirschfelder, Formal Rayleigh-Schrödinger perturbation theory for both degenerate and non-degenerate energy states, Internat. J. Quantum Chem. 3 (1969), 731-748.

[5] R. A. Horn and C. R. Johnson, Matrix Analysis, Cambridge University Press, Cambridge, 1990.

[6] T. Kato, Perturbation Theory for Linear Operators, Springer-Verlag, New York, 1980.

[7] M. Konstantinov, V. Mehrmann, and P. Petkov, Perturbed spectra of defective matrices, J. Appl. Math. 2003 (2003), no. 3, 115-140.

[8] B. N. Parlett, The Symmetric Eigenvalue Problem, Classics in Applied Mathematics, vol. 20, Society for Industrial and Applied Mathematics (SIAM), Pennsylvania, 1998.

[9] L. Rayleigh, The Theory of Sound. Vol. I, Dover Publications, New York, 1894.

[10] F. Rellich, Perturbation Theory of Eigenvalue Problems, Institute of Mathematical Sciences, New York University, New York, 1953.

[11] E. Schrödinger, Quantisierung als Eigenwertproblem. III, Ann. Physik 80 (1926), 437-490 (German).

[12] G. W. Stewart and J. G. Sun, Matrix Perturbation Theory, Computer Science and Scientific Computing, Academic Press, Massachusetts, 1990.

[13] J. H. Wilkinson, The Algebraic Eigenvalue Problem, Clarendon Press, Oxford, 1965.

Brian J. McCartin: Applied Mathematics, Kettering University, 1700 West Third Avenue, Flint, MI 48504-4898, USA

E-mail address: bmccarti@kettering.edu 


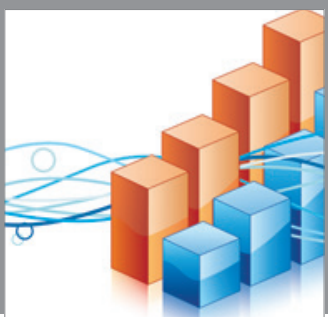

Advances in

Operations Research

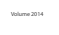

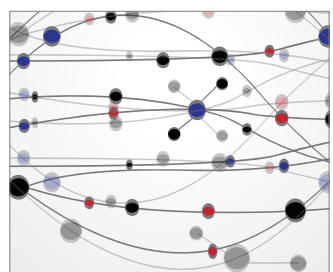

\section{The Scientific} World Journal
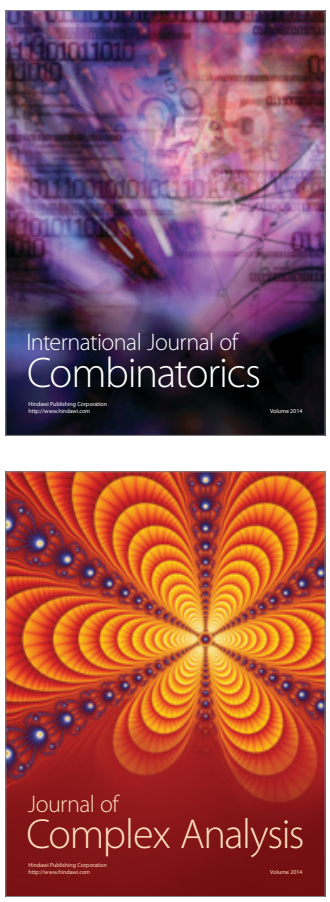

International Journal of

Mathematics and

Mathematical

Sciences
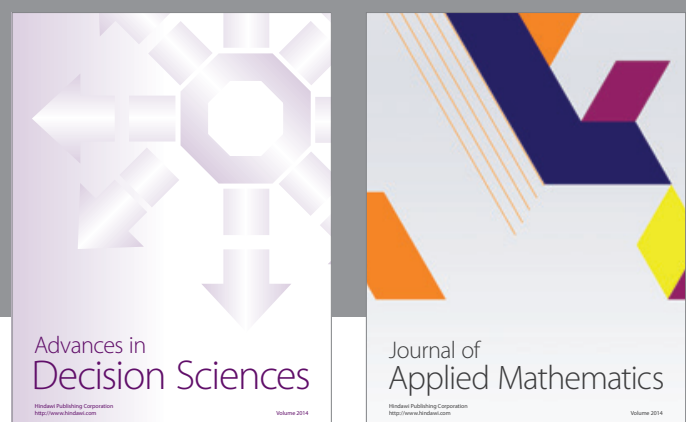

Journal of

Applied Mathematics
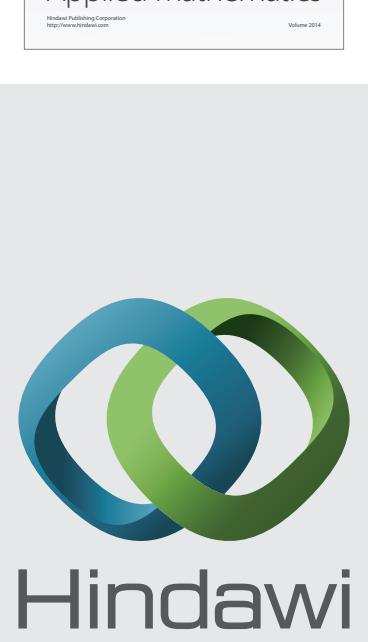

Submit your manuscripts at http://www.hindawi.com
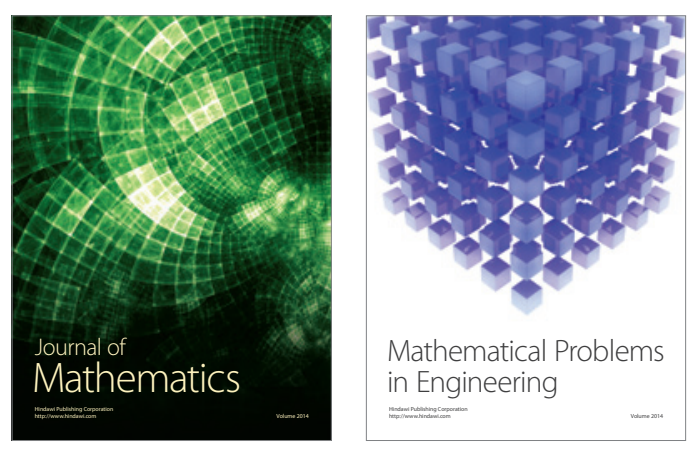

Mathematical Problems in Engineering
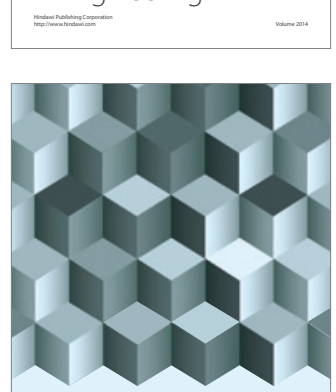

Journal of

Function Spaces
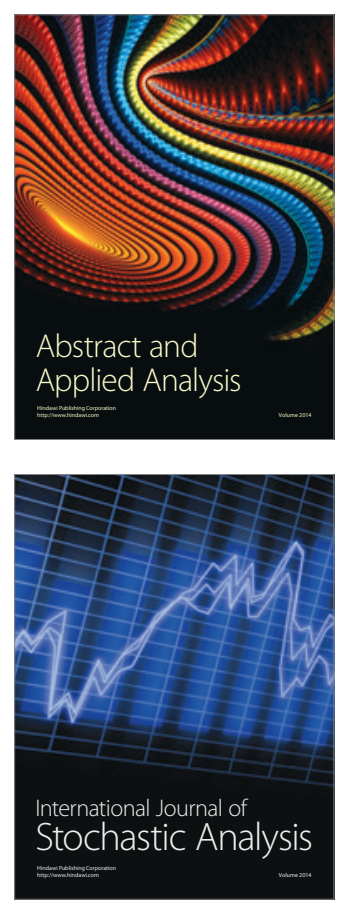

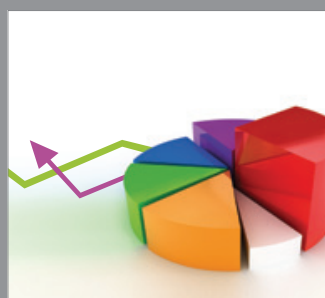

ournal of

Probability and Statistics

Promensencen
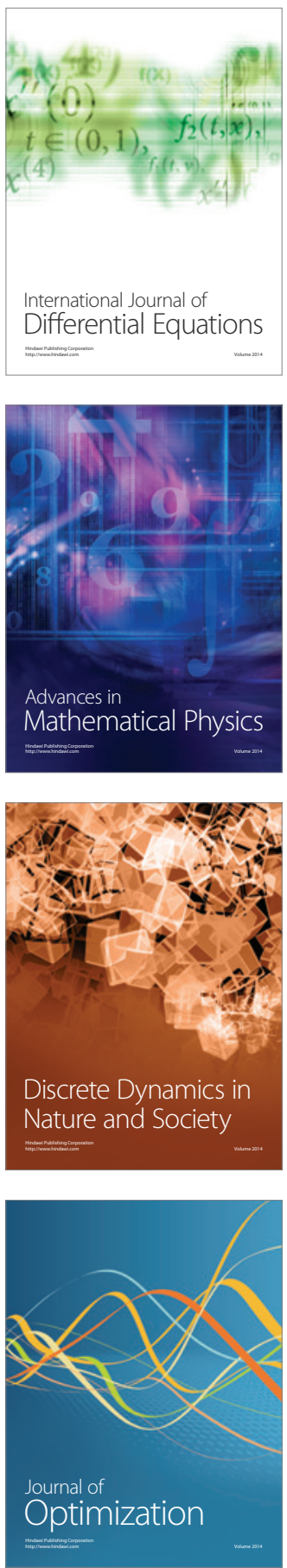